\title{
SVC PLACEMENT USING CRITICAL MODES OF VOLTAGE INSTABILITY
}

\author{
Yakout Mansour Wilsun Xu \\ B.C. Hydro \\ Burnaby, B.C., Canada
}

\author{
Fernando Alvarado \\ University of Wisconsin \\ Madison, Wisconsin, USA
}

\author{
Chhewang Rinzin \\ Department of Power \\ Bhutan
}

\begin{abstract}
The location of SVC (Static VAR Compensators) and other types of shunt compensation devices for voltage support is an important practical question. This paper considers a tool based on the determination of critical modes. Critical modes are computed by studying the system modes in the vicinity of the Point of Collapse. System participation factors for the critical mode are used to determine the most suitable sites for system reinforcement. Because the method does not rely on base case linearizations, the method is able to properly consider all system limits and nonlinear effects. The paper tests the proposed method by performing an assessment of the impact of the addition of Static VAR compensators to a 1380 bus model of the BC Hydro system.
\end{abstract}

\section{Introduction}

The location of SVC (Static VAR Compensators) and other types of shunt compensation devices for voltage support is an important practical question. This paper uses a tool based on critical modes. The most critical system mode can be computed by performing a Point of Collapse power flow solution, which requires the computation of the system Jacobian matrix at the point of maximum loadability [5]. The method amounts to solving a power flow problem subject to the constraint that the Jacobian of the power flow itself be singular [4, 14].

This paper considers an approximation to this approach that works well in practice: the system is loaded to near its point of collapse, and modal analysis is performed on this stressed system. One advantage of the pragmatic approach is that standard software can be used in the analysis, namely ordinary power flows and eigen-structure analysis programs $[7,10]$. The arguably more accurate notion of using the precisely determined

This paper was presented at the 1993 IEEE Power Industry Computer Application Conference held in Scottsdale, Arizona, May 4 - 7, 1993.
Point of Collapse [4] requires the use of more specialized software [5], and may form the basis for further refinements of the method.

The manner in which system nonlinearities are considered can have significant impact on the identification of critical modes. Base-case linearizations are generally not acceptable. The full nonlinear effect of components such as generators and SVCs must be taken into account in the identification of critical modes. The behavior of system components (and their impact on the system eigenvalues) can change drastically as limits are encountered.

Voltage itself is a poor indicator of proximity to system collapse conditions. Consider, for example, the voltage versus reactive power relationship for an idealized generator (Figure 1). The figure illustrates the difficulties associated with the linearization of a component that exhibits discontinuous behavior. A small change in demand can move the system from point "a" to point "b." If this generator is linearized at point "a," the slope of the $V$ vs. $Q$ is zero, indicating that the machine is of a $P V$ type and fully capable of regulating its voltage. If the generator is linearized at " $b$," the slope is $\infty$, indicating a machine of a $P Q$ type and unable to regulate its voltage. This drastic slope change can lead to very different results from modal analysis. Since components are likely to reach limits in the vicinity of the maximum loadability point, representation of these limits is important. In fact, the reaching of limits helps identify those system components most responsible for a critical mode. The Point of Collapse method fully accounts for all system nonlinearities and limits. If one does not wish to solve a problem exactly at its point of collapse, a reasonable alternative for the consideration of limits is to solve the problem near the collapse point and to use the notion of a "conversion margin" which can be either additive or multiplicative:

$$
\begin{gathered}
Q_{\text {actual }}-Q_{\text {margin }}>Q_{\text {limit }} \\
Q_{\text {actual }} \cdot Q_{\text {margin }}>Q_{\text {limit }}
\end{gathered}
$$

For cases where one does not solve the system precisely at the collapse point, a conversion margin $Q_{\text {margin }}$ enables a user to have some control on which slope to use in the modal analysis. 


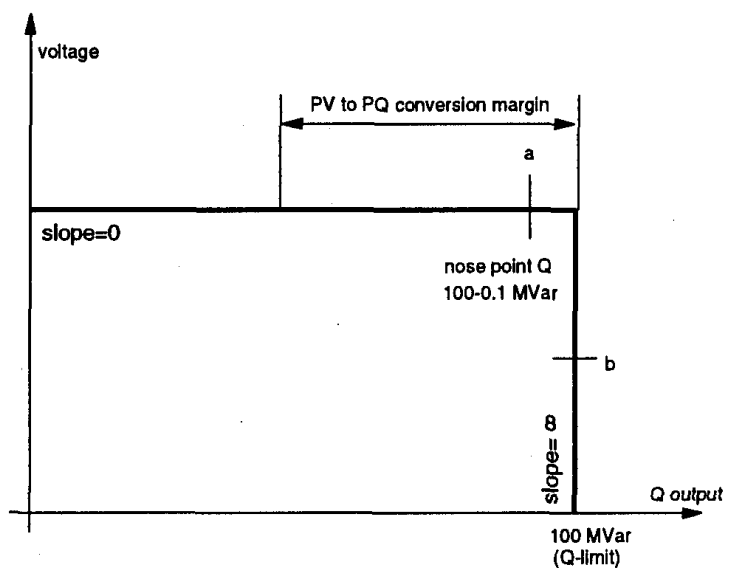

Fig. 1: Steady-state voltage versus reactive power relationship for a generator

\section{Modal analysis}

Modal or eigenvalue analysis of the system Jacobian matrix near the point of voltage collapse can be used to identify buses vulnerable to voltage collapse and locations where injections of power benefit the system most. When modal analysis is used, there is no need to drive the system precisely to its "nose point" to ensure that a maximum level of stress is reached. The eigenvectors of the critical eigenvalue give information about the loads responsible for the voltage collapse.

For a single load power system, the linearized relationship between load voltage and reactive power load can be described by the following equation:

$$
\lambda \Delta V=\Delta Q
$$

where $\lambda$ is the $V-Q$ sensitivity. In general, this sensitivity is obtained from the network Jacobian matrix. When $\lambda$ is close to zero, a small load change results in a large voltage change. The operating point is considered to be voltage unstable from the load flow point of view.

Consider a system whose Jacobian matrix is diagonal:

$$
\left[\begin{array}{cccc}
\lambda_{1} & 0 & \cdots & 0 \\
0 & \lambda_{2} & \cdots & 0 \\
\vdots & \vdots & \ddots & \vdots \\
0 & 0 & \cdots & \lambda_{n}
\end{array}\right]\left[\begin{array}{c}
\Delta V_{1} \\
\Delta V_{2} \\
\vdots \\
\Delta V_{n}
\end{array}\right]=\left[\begin{array}{c}
\Delta Q_{1} \\
\Delta Q_{2} \\
\vdots \\
\Delta Q_{n}
\end{array}\right]
$$

If $\lambda_{1}$ is close to zero, a small load change at bus 1 would result in a large change in the voltage at the bus. However, all other buses would be unaffected. Clearly, voltage collapse would happen at bus 1 . Unfortunately, the Jacobian matrices of actual power systems are not diagonal matrices. However, they can be diagonalized using modal analysis. With this technique, one obtains the following matrix relationship between modal volt- ages $\Delta v$ and modal injections $\Delta q$ :

$$
\left[\begin{array}{cccc}
\lambda_{1} & 0 & \cdots & 0 \\
0 & \lambda_{2} & \cdots & 0 \\
\vdots & \vdots & \ddots & \vdots \\
0 & 0 & \cdots & \lambda_{n}
\end{array}\right]\left[\begin{array}{c}
\Delta v_{1} \\
\Delta v_{2} \\
\vdots \\
\Delta v_{n}
\end{array}\right]=\left[\begin{array}{c}
\Delta q_{1} \\
\Delta q_{2} \\
\vdots \\
\Delta q_{n}
\end{array}\right]
$$

In this equation the modal voltages and modal injections are obtained from $[\Delta v]=[T][\Delta V]$ and $[\Delta q]=$ $[T][\Delta Q]$ where $[T]$ is the modal matrix of eigenvectors. The vectors $[\Delta v]$ and $[\Delta q]$ have the same units as the actual vectors. They are linear combinations of the actual physical voltages and injections. As $\lambda_{1} \rightarrow 0$, modal voltage 1 becomes very sensitive to modal injection 1 while other modes remain unaffected.

The main conclusion from this is that voltage collapse is actually the collapse of a modal voltage. That is, the power system cannot support a particular combination of reactive power loads. If modal voltages are plotted as a function of system stress, only one modal voltage experiences collapse. However, since several (and to some degree, all) physical voltages participate to a greater or lesser degree on the mode in question, this causes multiple physical voltages to collapse.

The participation of each load in the critical mode determines the importance of the load in the collapse [6]. The degree of participation is determined from an inspection of the entries of the left eigenvector of the critical mode. Larger eigenvector entries signify locations most suitable for voltage support or load shedding.

\section{Modal Transformation of the Reduced System Jacobian}

Eigenanalysis $[8,11,12]$ uses a linearization of the non-linear steady state power system equation $f(x, \lambda)=0$ :

$$
\left[\begin{array}{l}
\Delta P \\
\Delta Q
\end{array}\right]=\left[\begin{array}{cc}
H & N \\
M & L
\end{array}\right]\left[\begin{array}{c}
\Delta \theta \\
\Delta V
\end{array}\right]
$$

This equation is obtained as the steady-state of a dynamic model that considers also the impact of the dynamics of all generators and generator controls. For the time frames of interest in voltage collapse, this model can effectively be a constant terminal voltage voltage provided one is assured that faster modes are stable. As in power flow solutions, voltage-dependent load models can be included in the modal analysis. Steadystate load-voltage characteristics should be used, since the modal analysis deals with the steady-state conditions of the system. This is valid for modal analysis of the critical mode near the voltage collapse bifurcation. The steady-state load-voltage characteristics are different from transient load-voltage characteristics adopted for transient stability simulations. For further information on load models appropriate to voltage collapse studies refer to $[16,9]$. 
Although both $P$ and $Q$ changes affect system conditions, it is possible to study the approximate effects of reactive power injections on voltage stability by setting $\Delta P=0(P$ constant $)$ and deriving the $Q-V$ sensitivities at different loads. Using arguments similar to those of [14], an approximate equation 6 can be rewritten as:

$$
\Delta Q=\left[L-M H^{-1} N\right] \Delta V=J_{R} \Delta V
$$

The matrix $J_{R}$ in equation 7 is the reduced Jacobian and it relates voltage and reactive power at each bus.

The smallest eigenvalue, which at the saddle node bifurcation becomes zero is calculated by performing a modal transformation of the reduced Jacobian matrix of the system as:

$$
J_{R}=\xi \Lambda \eta
$$

where $\xi$ and $\eta$ are the left and right eigenmatrices respectively and $\Lambda$ represents the system eigenvalues. The components of the left eigenvector can be interpreted as indicating a direction normal to the operational boundary of the system $[6,13]$. Right eigenvector components indicate the degree to which given variables are involved in a given mode. The use of both left and right eigenvector information leads to the notion of participation factors. This paper uses bus participation factors, which are defined for bus $k$ and critical mode $i$ as $\xi_{k i} \eta_{i k}$.

Identifying the critical mode eigenvalue, $\lambda_{i}$, is not trivial. The magnitude of an eigenvalue is only a relative measure of proximity to collapse [8]. As the system approaches collapse, eigenvalues that initially have small real component may not be critical, and other eigenvalues may become critical. Proximity of an eigenvalue to the imaginary axis is not sufficient. A methodology to identify the critical mode is presented next.

\section{Stressing the system}

Based on earlier discussions, it is evident that modal analysis must be done on a stressed system. Stressing a system all the way to its point of collapse was not considered a practical approach using an available production grade power flow type program. Instead, this paper performs the determination of the critical mode in the vicinity of the maximum loadability point. In this application, the real and reactive power loads at the buses in the area of interest are scaled up according to predetermined weighting factors. These predetermined weighting factors can correspond to a known expected direction of demand increase. Although different directions can identify different critical modes, in practice the approach has proven to be robust in the identification of critical modes irrespective of the exact direction chosen, particularly when the procedure for the elimination of local modes described later in this section is used. Finally, a procedure has been recently developed for the computation of the worse possible demand increase direction [2].

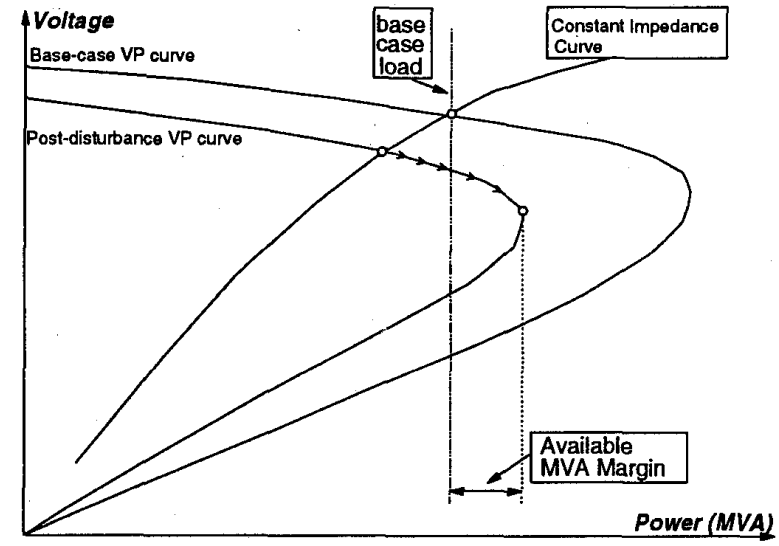

Fig. 2: VP nose curve for base case and contingency.

The load increase is done in many steps until the nose of the $P-V$ curve is reached. While the technique is simple in concept, its implementation involves many details. In this regard, reference to continuation power flows is useful $[1,5]$. At the maximum loadability point, the system must satisfy both the power flow equations and a condition for the singularity of the $\mathrm{Ja}$ cobian matrix. Mathematically, this corresponds to the simultaneous solution of the power flow equations augmented with a matrix equation of the form $J_{R} y=0$ with $|y| \neq 0$. In practice, exact solution for this point of collapse is not necessary. The expedient procedure of iterating toward the maximum loadability point with ever decreasing step sizes yields results within 2.0 MW of the correct solution within a few iterations. Like the continuation power flow, this method is capable of taking into account all system limits with the added advantage of using ordinary computational tools.

The main problem with the solution process concerns the solvability of a stressed case after a disturbance. One practical way to resolve the problem and compute accurate MVA margins is depicted in figure 2. The base-case is solved up to a certain load level. At this point, a discrete disturbance such as the outage of a heavily loaded transmission line is applied. In order to render this system solvable, load models are converted momentarily to constant impedance models, enabling the system to be readily solvable along the constant impedance curve. After a post-disturbance solution is obtained, the load models are modified once more and the same stressed-system power flow solver as before is applied. This approach resembles the response of loads in the period following a disturbance [16]. The reason that a constant power load is a reasonable model for power loads is due in part to the action of tap changers that isolate demands from transmission-side voltage variations. Thus, an impedance load behaves initially as a constant impedance, and only after tap changer action does it behave as a constant power load. 


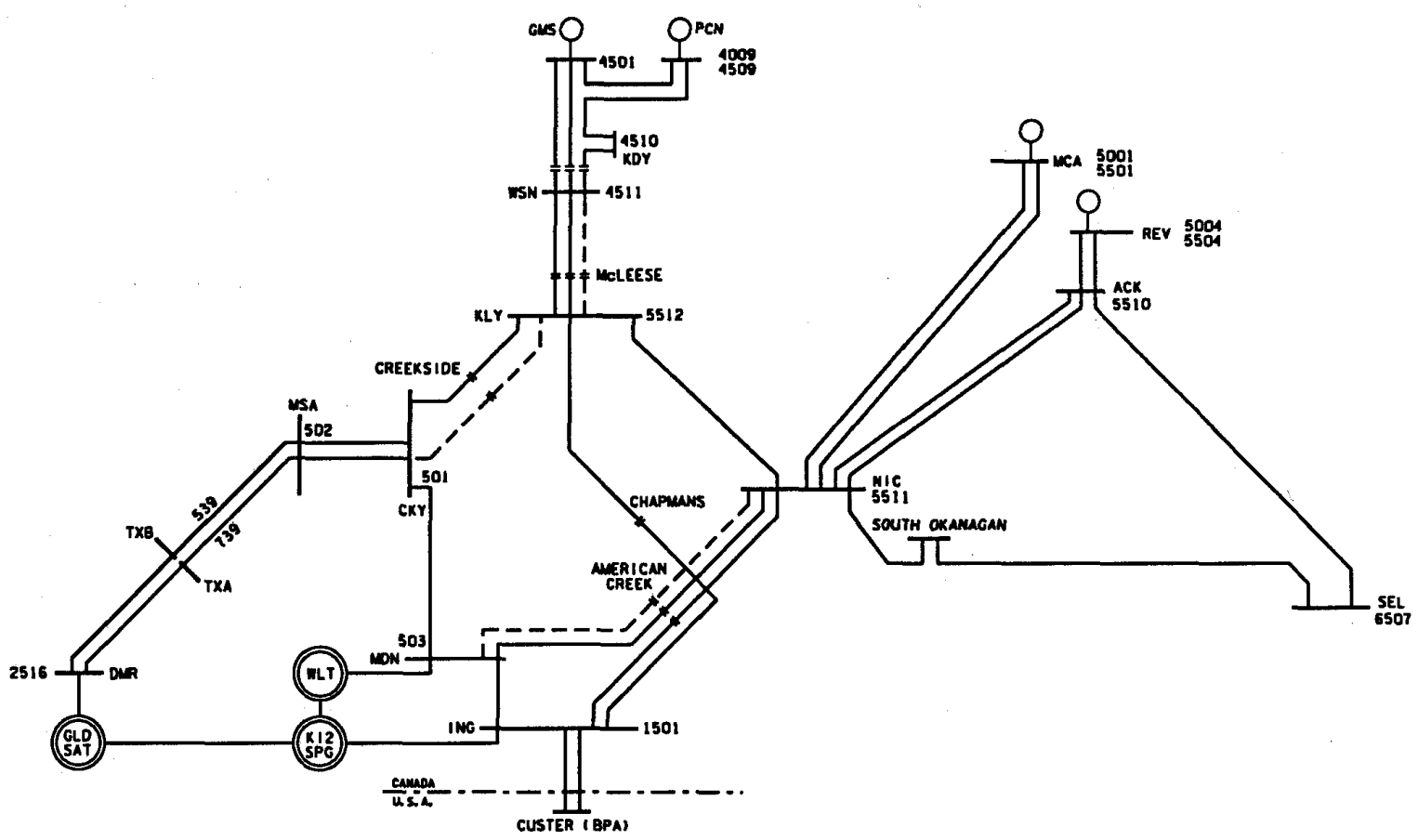

Fig. 3: Relevant portion of 1380 bus BC Hydro System model

Allocation of power among generators is another issue. Generator outputs are scheduled in proportion to their remaining output capability. However, other generation scheduling methods can be explored [3].

Other features of the stressed power flow method used during these tests include: power can be scaled by area, zones and individual buses, both their active and reactive components.

Once the system has been stressed, the objective is to identify the critical modes. This is done by performing modal analysis on the last solved case. A positive eigenvalue indicates that the maximum loadability point is beyond the bifurcation point for the system. The critical mode is the mode associated either with this eigenvalue or with one of the small real eigenvalues. In general it is useful to track three or four eigenvalues. As a result of linearization of the control equations, it may be the case that the critical mode may not be the one that initially appears to be critical based on the location of the eigenvalue at base case conditions.

Eigenvalues with few participating buses correspond to local modes. These normally reveal local voltage problems such as problems resulting from a long line feeding a remote load. Alleviating these modes by local means (adding reactive power) and proceeding with the stressing of the system eventually will reveal system modes. While in an operations context both local and system modes are quite important, in a planning context local modes are somewhat easier to remedy by local means. Thus it is of interest, in addition to find- ing all important local modes, to find the most important system modes. Modes with small eigenvalue real parts and large numbers of participating buses reveal system-wide or area-wide problems. This procedure for the identification of important system modes by explicit removal of local modes has been found to be more valuable than the notion of identifying modes by tracking multiple modes with small real eigenvalue components.

The combined use of system-wide stress and modal analysis can provide reliable information for the study of voltage stability problems. The next section illustrates an application to the design of system reinforcements.

\section{Application of Modal Analysis to Planning}

The technique described in the previous sections has been applied to planning of a full-scale version of the BC Hydro (BCH) System. Figure 3 illustrates the relevant portion of this system. The main concerns in this study were the delivery of power to the main load centers: the Lower Mainland (in the vicinity of bus ING) and Vancouver Island (in the vicinity of buses GLD and SAT), whichever was more likely to collapse, and to determine the most effective location for the placement of voltage support. This section presents some results from these studies.

The first study checks if participation factors can predict the voltage collapse area. For this purpose, bus ING (key bus in Lower Mainland) and bus SAT (key 


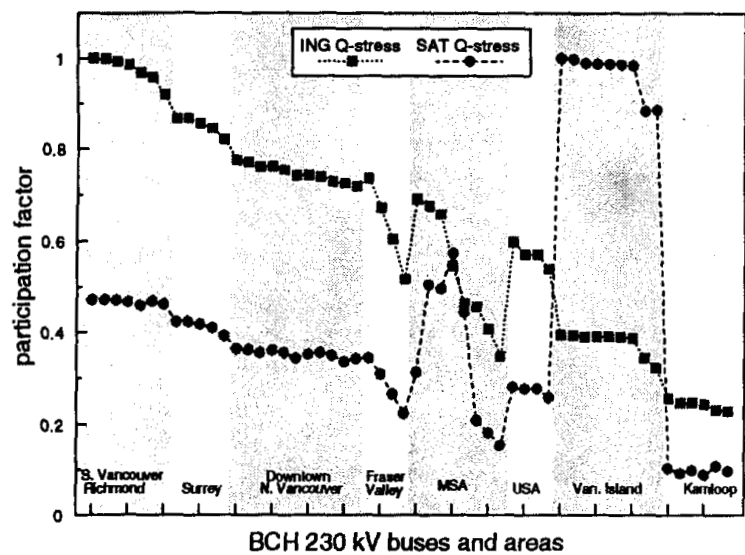

Fig. 4: Critical mode eigenvalue participation factors for demand increase at ING and SAT.

bus in Vancouver Island) are stressed with increased reactive power load respectively. The near-nose points computed with the stressed power flow are then analyzed using modal analysis. Figure 4 illustrates the participation factors for all $230 \mathrm{KV}$ buses. A large participation factor value indicates a high involvement of the bus in the voltage collapse. It can be seen that the Lower Mainland buses have large participation factors when ING is stressed. If SAT is stressed, the Vancouver Island buses have high participation factors. The usefulness of the modal information is thus demonstrated.

If the entire $\mathrm{BCH}$ system is stressed with a uniform MVA load increase as specified in the planning criteria, identifying the critical areas with intuition becomes impossible. Modal analysis has to be used. Figure 5 shows the participation factors near the nose point when the entire BCH system is stressed according to the planning criteria. The Lower Mainland is found to be the critical area. This area can be further divided into several sub-areas. Among them, the Vancouver-south is the most critical one and is probably the best location for effective voltage support.

Six $230 \mathrm{kV}$ buses are then selected as candidate SVC locations based on figure 5 . The SVC size, $\pm 150 \mathrm{MVar}$, is determined based on the need to continue to meet the $\mathrm{BCH}$ voltage stability planning criteria for some time after the SVC is put into operation [15]. The effectiveness of a SVC is evaluated according to the resulting improvement on system margin, both present and future. Different SVC locations yield different margin improvements, as illustrated in Figure 6. Observations from this figure include:

- The best SVC locations are buses SPG or KI2, which are in the center of the critical area identified by the participation factors.

- There is a good correlation between margin improvement and participation factors.

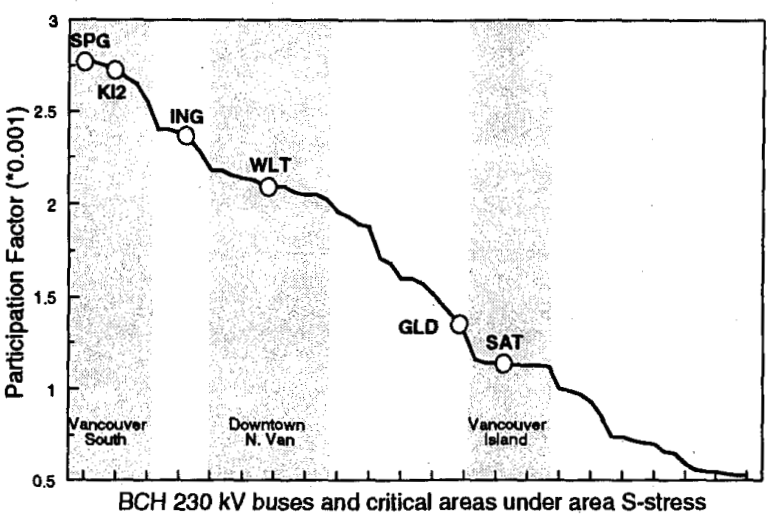

Fig. 5: Identification of critical areas in BCH System.

Modal analysis was also performed for cases with SVC installed at buses WLT and SAT. Participation factors (both before and after the addition of SVC compensation) for these two cases are shown in figure 7 . The figure indicates that with a SVC at WLT the participation factors, and therefore the risks of voltage collapse around the WLT area, are reduced. A similar conclusion applies to the SAT case.

The SVC used in this study represents a general shunt compensation device. It is intended to demonstrate the effectiveness of the proposed method. The speed with which the shunt device should operate is a separate issue. This problem involves dynamic aspects of the voltage collapse phenomenon, and cannot be properly analyzed using modal analysis methods based on power flow static models. Detailed studies of the rate of voltage collapse and the response time requirements for reactive power support can be found in [16].

A concern not properly addressed by the steady-state methodology in this paper is the situation where the system is safely operable both before and after the outage of a component, but where the sudden transition itself can cause an instability. This mode of failure is the dominant mode of failure for angular instability. It has been assumed that this is not expected to be the case for voltage stability problems.

\section{Conclusions}

Modal analysis in the vicinity of the point of collapse of a system can be a very valuable tool that permits the direct comparison of alternatives. This paper has shown that, if some care is taken, power flow programs and eigenvalue analysis packages can be used to study the system in the vicinity of these points. The paper describes a comprehensive methodology for the identification of system weaknesses in a systematic manner which is both theoretically sound and validated with practical design experience. 
Acknowledgement

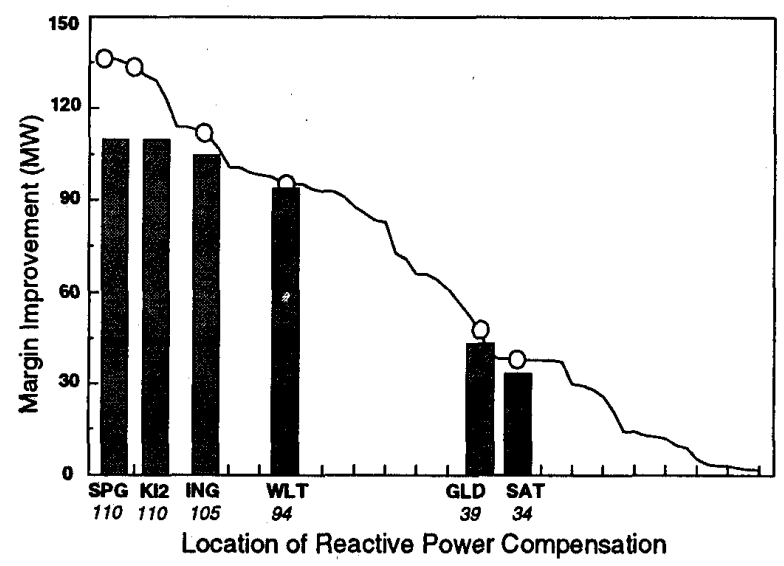

Fig. 6: Effect of SVC location on margin improvement. Bars (and numeric values below them) indicate margin improvement. The critical mode participation factors are illustrated as background to demonstrate correlation between margin improvement and participation factor.

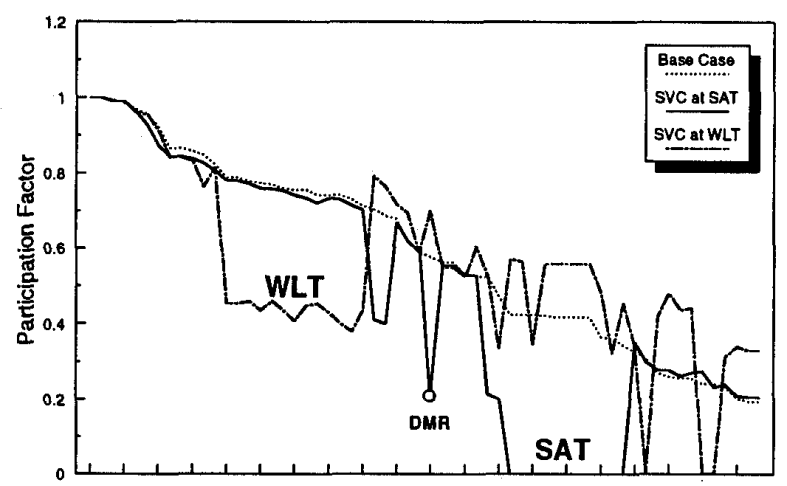

$\mathrm{BCH} 230 \mathrm{kV}$ bus and critical area

Fig. 7: Effect of SVC location on area voltage stability. After the addition of compensation, the base case participation factors (and consequently the risk of collapse) for buses in the respective compensated areas are significantly reduced.
The first two authors acknowledge the support of BC Hydro. The third author acknowledges the support of the Electric Power Research Institute under the supervision of Rambabu Adapa. The fourth author acknowledges the support of the Royal Government of Bhutan.

\section{REFERENCES}

[1] V. Ajjarapu and C. Christy. The continuation power flow: A tool for steady state voltage stability analysis. In Power Industry Computer Applications Conference, pages 304-311, May 1991.

[2] F. L. Alvarado, I. Dobson, and Y. Hu. Computation of closest bifurcations in power systems. Technical report, Electrical and Computer Engineering, The University of Wisconsin, February 1993.

[3] F. L. Alvarado and Y. Hu. Dispatch policy effects in the determination of system security. In NSF Workshop on The Impact of a Less Regulated Environment on Power System Control and Security, pages 53-77, Madison, Wisconsin, April 1991.

[4] F. L. Alvarado and T. H. Jung. Direct detection of voltage collapse conditions. In Proceedings of the International Conference on Bulk Power System Voltage Phenomena - Voltage Stability and Security, pages 5.23-5.38, Trout Lodge, Potosi, Missouri, September 18-24, 1988.

[5] C. A. Cañizares and F. L. Alvarado. Point of collapse and continuation methods for large AC/DC systems. Presented at the IEEE/PES Winter Meeting, January 1992.

[6] I. Dobson. Observations on the geometry of saddle node bifurcation and voltage collapse in electric power systems. IEEE Transactions on Circuits and Systems, Part 1, 39(3):240-243, March 1992.

[7] D. M. Elwood. Eigenanalysis of large electric power systems. Technical Report PNL-7632, Batelle Pacific Northwest Laboratory, February 1991. Available from NTIS, contract DE-AC06-76RLO 1830.

[8] B. Gao, G. K. Morison, and P. Kundur. Voltage stability evaluation using modal analysis. IEEE Transactions on Power Systems, 7(4), November 1992.

[9] D. J. Hill. Nonlinear dynamic load models with recovery for voltage stability studies. IEEE Transactions on Power Systems, 1992. To appear.

[10] P. Kundur, G. J. Rogers, and D. Y. Wong. Small signal stability analysis program package - version 2.0 user manual. Technical report, EPRI, Palo Alto, CA, 1990. Report number EPRI EL-6678.

[11] I. J. Perez-Arriaga, G. C. Verghese, and F. Schweppe. Selective modal analysis with applications to electric power systems, part i: Heuristic introduction. IEEE Transactions on Power Apparatus and Systems, 101:3117-3215, 1982.

[12] I. J. Perez-Arriaga, G. C. Verghese, and F. Schweppe. Selective modal analysis in power systems. Technical report, EPRI, 1983.

[13] Chhewang Rinzin, Fernando L. Alvarado, and Rambabu Adapa. Formulas for geographic differentiation of security limits. In 24th North American Power Symposium, October 1992. 
[14] T. VanCutsem. A method to compute reactive power margins with respect to voltage collapse. IEEE Transactions on Power Systems, 6(2):145-156, February 1991.

[15] Working group on Voltage Stability. Voltage stability of power systems: Concepts, analytical tools and industry experience. Technical report, IEEE, 1990.

[16] W. Xu and Y. Mansour. Voltage stability analysis using generic dynamic load models. Presented at the IEEE/PES Winter Meeting, Columbus, Ohio, paper No. 185-9-PWRS, 1993.

Yakout Mansour (SM) obtained his B.Sc. degree from the University of Alexandria, Egypt in 1971 and the M.Sc. degree from the University of Calgary, Canada in 1977, both in electrical engineering. His work experience includes utility industry in Egypt, consulting with' Montreal Engineering Co. in Calgary, Alberta, Canada. He joined B.C. Hydro as a transmission planning engineer in 1978, and progressed to a senior analytical studies engineer, then manager of Power Engineering at Powertech (a B.C. Hydro subsidiary) then to his present capacity as manager of Regional System Planning Department, B.C. Hydro. He is chairman of the IEEE Working Group on Voltage Stability.

Wilsun (Wenyuan) $\mathrm{Xu}$ obtained a B.Eng. degree from Xian Jiaotong University, China, a M.Sc. degree from the University of Saskatchewan and a Ph.D. from the University of British Columbia, all in electrical engineering. He currently works as an analytical study engineer in the Regional System Planning Department of B.C. Hydro and is involved in power system harmonics and voltage stability studies.

Fernando L. Alvarado (F'93) obtained a Ph.D. from the University of Michigan. He is currently a Professor at the University of Wisconsin in Madison in the Department of Electrical and Computer Engineering. His main interests are in computer applications to power systems and large scale problems.

Chhewang Rinzin obtained his B.S. and M.S. degrees from the University of Wisconsin in Madison in 1991 and 1992 respectively. He spent a summer internship with B.C. Hydro in 1991. He is presently an engineer with the Department of Power, Royal Government of Bhutan. 